\title{
How to Improve Urban Intelligent Traffic? A Case Study Using Traffic Signal Timing Optimization Model Based on Swarm Intelligence Algorithm
}

\author{
Xiancheng Fu ${ }^{1}$, Hengqiang Gao ${ }^{1}{ }^{*}$, Hongjuan Cai ${ }^{2}$, Zhihao Wang ${ }^{3}$ and Weiming Chen ${ }^{3}$ \\ 1 School of Mechanical Engineering and Electronic Information, China University of Geosciences, \\ Wuhan 430074, China; fuxiancheng_cug@outlook.com \\ 2 School of Information Science and Engineering, Wuchang Shouyi University, Wuhan 430064, China; \\ caihongjuan_cug@outlook.com \\ 3 Faculty of Engineering, China University of Geosciences, Wuhan 430074, China; \\ wzh_gcxy@cug.edu.cn (Z.W.); chenweiming@cug.edu.cn (W.C.) \\ * Correspondence: gaohengqiang@outlook.com
}

check for updates

Citation: Fu, X.; Gao, H.; Cai, H.; Wang, Z.; Chen, W. How to Improve Urban Intelligent Traffic? A Case Study Using Traffic Signal Timing Optimization Model Based on Swarm Intelligence Algorithm. Sensors 2021, 21, 2631. https://doi.org/10.3390/ s21082631

Academic Editors: Enrique Alba and Zakaria Abdelmoiz Dahi

Received: 29 March 2021

Accepted: 6 April 2021

Published: 8 April 2021

Publisher's Note: MDPI stays neutral with regard to jurisdictional claims in published maps and institutional affiliations.

Copyright: (C) 2021 by the authors. Licensee MDPI, Basel, Switzerland. This article is an open access article distributed under the terms and conditions of the Creative Commons Attribution (CC BY) license (https:// creativecommons.org/licenses/by/ $4.0 /)$.

\begin{abstract}
Traffic congestion is a major problem in today's society, and the intersection, as an important hub of urban traffic, is one of the most common places to produce traffic congestion. To alleviate the phenomenon of congestion at urban traffic intersections and relieve the traffic pressure at intersections, this paper takes the traffic flow at intersections as the research object and adopts the swarm intelligent algorithm to establish an optimization model of intersection traffic signal timing, which takes the average delay time of vehicles, the average number of stops of vehicles and the traffic capacity as the evaluation indexes. This model adjusts the intersection traffic signal timing intelligence according to the real-time traffic flow and carries out simulation experiments with MATLAB. Compared with the traditional timing schemes, the average delay time of vehicles is reduced by $10.25 \%$, the average number of stops of vehicles is reduced by $24.55 \%$, and the total traffic capacity of the intersection is increased by $3.56 \%$, which verifies that the scheme proposed in this paper is effective in relieving traffic congestion.
\end{abstract}

Keywords: intersection; traffic signal timing; labor division; real-time control

\section{Introduction}

The collective behavior of social insects through simple cooperation is called "swarming intelligence (SI)". In 1992, Dorigo [1] proposed the ant colony algorithm (ACO) in the swarm intelligence algorithm. Then, Levy [2] first introduced swarm intelligence in detail in 1994. After that, particle swarm optimization (PSO) [3], artificial fish swarm algorithm (AFSA) [4], artificial bee colony algorithm (ABC) [5], and other algorithms based on biological foraging were proposed by scholars, and the research on swarm intelligence was gradually deepened. With the development of the information age, those algorithms have been widely used in various kinds of fields, including the public opinion polarization process [6-9], fall detection [10], analysis of user satisfaction [11], welding flame detection [12], and road networks $[13,14]$.

Division of labor is also an important behavior of swarm intelligence [15], which has been widely used in task allocation [16,17], space allocation [18,19], robots, and Unmanned Aerial Vehicle (UAV) [20,21] in recent years.

In recent years, with the continuous improvement of people's living standards, the traffic demand is also increasing, and traffic congestion has become a major problem faced by many cities. Because urban traffic is a complex system with many controlled variables [22], the urban traffic problem has become a "perennial difficulty" among many urban problems. For this reason, scholars have put forward various countermeasures and applied a variety of emerging technologies. Hakim et al. [23] proposed a fuzzy Technique 
for Order Preference by Similarity to an Ideal Solution (TOPSIS) algorithm based on the realtime number and classification of vehicles, which could set the priority vehicles according to the length of vehicles, and applied this algorithm in the design of the timing control system of traffic signal lights. Guo et al. [24] proposed a timing strategy including green time optimization and lane combination calculation. According to the real-time traffic flow, this strategy can optimize the greening time, calculate the lane combination, adjust the cycle, and get the timing plan. The experiment proves that this strategy can improve the traffic efficiency of the intersection by more than $15 \%$. Chen et al. [25] proposed a traffic signal light control system based on model predictive control (MPC), which is divided into the traffic flow prediction model and the traffic timing optimization method. On the basis of the automatic collection of traffic flow data, appropriate time settings are obtained through the system to alleviate traffic congestion. Taking the cellular transmission model as the basic model, Qi [26] proposed an optimal timing model for a multi-intersection signal control scheme according to the characteristics of a multi-intersection road network, combined with control precision and real-time requirements. The model can adapt to the change of traffic flow at the intersection through time adjustment, and reduce the average delay time of road vehicles.

This paper will do some exploratory research on the application of the ant colony labor division model to solve the problem of traffic signal timing of the intersection. With taking the real-time traffic condition of the intersection as the research object, the optimization model of traffic signal timing of the intersection is established based on the evaluation index of the delay time of vehicles, the number of stops of vehicles, and the traffic capacity. This model is able to adjust the traffic signal timing intelligently according to real-time traffic conditions, reduce the conflict between traffic flows and alleviate the phenomenon of urban traffic congestion.

\section{Ant Colony Labor Division Model}

\subsection{Basic Ant Colony Labor Division Model}

Social insects will take the form of division of labor to complete tasks, and assign various tasks to different individuals. On the basis of an in-depth study of ant colony labor division behavior, Bonabeau et al. [27] proposed a fixed response threshold model, which believed that ant colony labor division behavior originated from the different responses of individual internal threshold to external stimuli. The stimulus value of the external task represents the urgency of the task, and the stimulus value varies with time and the number of ants currently performing the task. The internal threshold of an individual represents the tendency of the individual to perform the task, and the level of threshold is determined by the individual itself. When the stimulus value of the external task is far more than the internal threshold of the ant, the probability of the individual to perform the task is high. Otherwise, the probability of the individual to perform the task is low.

This paper uses $s$ to represent the stimulus value of the external task, $\theta$ to represent the internal response threshold of an ant, $\Delta$ to represent the increase of the stimulus value of per unit time, $\alpha$ to represent the efficiency of the ant to perform the task, and $N_{\text {act }}$ to represent the number of ants currently performing tasks. Then, in each unit time, the probability of ants performing the task is shown in Equation (1), and the change of external task stimulus is shown in Equation (2). In the study of Castello et al. [28], $n$ represents a constant that controls the curve shape of the individual response threshold function, which is generally set as $n=2$.

$$
\begin{gathered}
P=\frac{s^{n}}{s^{n}+\theta^{n}} \\
s(t+1)=s(t)+\Delta-\alpha N_{a c t}
\end{gathered}
$$

\subsection{Optimization of Ant Colony Labor Division Model}

In the basic ant colony labor division model, ants will perform tasks in which environmental stimulus intensity exceeds their internal response threshold. In the traffic signal 
timing problem, different signal phases will increase or decrease the green time according to the traffic flow of each phase. Therefore, in the traffic signal timing problem, the signal phase can be regarded as different ants, and the traffic flow and green time of each phase can be regarded as the stimulus value of the external environment.

Here are the definitions of some of the notation involved in this model:

$q_{i}$ - the traffic flow at phase $i$;

$t_{g}{ }^{i}$ - the green time at phase $i$;

$s_{i}{ }^{+}$- the stimulus value of increasing green time at phase $i$;

$s_{i}{ }^{-}$- the stimulus value for the reduction of green time at phase $i$;

$\theta_{i}{ }^{+}$- the response threshold of increasing the green time in phase $i$;

$\theta_{i}{ }^{-}$- the response threshold of reducing the green time in phase $i$;

$P_{i}{ }^{+}$- the selection probability of the green time increase in phase $i$;

$P_{i}^{-}$- the selection probability of the green time decrease in phase $i$;

$P_{i}^{*}$-the selection probability of the green time invariant in phase $i$;

$\xi_{i}{ }^{+}$- the increment of green time for phase $i$;

$\xi_{i}{ }^{-}$- the reduction of green time for phase $i$;

$\lambda_{i}$ - the green ratio in phase $i$;

$x_{i}$ - the effective green time of phase $i$;

$y_{i}$ - the degree of traffic flow saturation;

$C$ - the cycle time;

$D_{i}$ - the delay time of vehicles in phase $i$;

$H_{i}$ - the number of stops of vehicles in phase $i$;

$Q_{i}$ - the traffic capacity in phase $i$.

\subsubsection{Environmental Stimulus}

In the problem of green time allocation at a traffic intersection, if the total cycle time is fixed, when the green time of a certain phase increases, the traffic congestion at that phase is relieved, but it will inevitably lead to the decrease of green time of other phases and the increase of the probability of traffic congestion. Generally speaking, in the distribution problem, when the interest subjects increase their interests, their willingness to continue to increase will be relatively weakened, and the willingness to reduce the interests will be relatively enhanced. On the contrary, when the interest subject reduces their interests, their willingness to continue to reduce will be relatively weakened, and the willingness to increase the interests will be relatively enhanced.

Let the current traffic flow at phase $i$ of the intersection be $q_{i}$ and the green time at phase i be $t_{g}{ }^{i}$. The stimulus value of increasing green time at this phase is $s_{i}{ }^{+}$, and the change of $s_{i}{ }^{+}$is shown in Equation (3). As can be seen from Equation (3), when $t_{g}{ }^{i}$ remains constant, the higher $q_{i}$ is, the higher $s_{i}{ }^{+}$is, indicating that the busier the current traffic flow is, the stronger the stimulus of increasing the green time of phase $i$ is. Similarly, when $q_{i}$ remains constant, the lower $t_{g}{ }^{i}$ is, the higher $s_{i}{ }^{+}$is, indicating that the shorter the current green time is, the stronger the stimulus of increasing the green time in phase $i$ is.

$$
s_{i}^{+}=\frac{q_{i}}{q_{i}+t_{g}^{i}}
$$

The stimulus value for the reduction of green time in phase $i$ is $s_{i}{ }^{-}$, and the change of $s_{i}{ }^{-}$is shown in Equation (4). As can be seen from Equation (4), when $q_{i}$ remains unchanged, the higher $t_{g}{ }^{i}$ is, the higher $s_{i}{ }^{-}$is, indicating that the longer the current green time is, the stronger the stimulus of reducing green time in phase $i$ is. Similarly, when $t_{g}{ }^{i}$ remains unchanged, the lower $q_{i}$ is, the higher $s_{i}{ }^{-}$is, indicating that the lower traffic flow is, the stronger the stimulus of reducing green time in phase $i$ is.

$$
s_{i}^{-}=\frac{t_{g}^{i}}{q_{i}+t_{g}^{i}}
$$




\subsubsection{Response Threshold}

In the process of green time allocation at traffic intersections, the busier the traffic flow of a phase is, the greater the tendency of green time increase of the phase is. On the contrary, the smaller the traffic flow of a phase is, the greater the tendency to reduce the green time at this phase is, and the smaller the tendency to increase it is.

$$
\begin{aligned}
\theta_{i}^{+} & =\frac{1}{k q_{i}} \\
\theta_{i}^{-} & =k q_{i}
\end{aligned}
$$

where $\theta_{i}{ }^{+}$is the response threshold of increasing the green time in phase $i$, and $\theta_{i}^{-}$is the response threshold of reducing the green time in phase $i$.

Equations (5) and (6) show that $\theta_{i}{ }^{+}$is inversely proportional to $q_{i}$. That is, when the traffic flow of phase $i$ is busier, the response threshold of green time increase in this phase is lower, and the tendency of green time increase is greater. $\theta_{i}{ }^{-}$is proportional to $q_{i}$. That is, when the traffic flow in phase $i$ is lower, the response threshold of green time reduction in this phase is lower, and the tendency of green time reduction is greater.

\subsubsection{Probability of Task Execution}

In the process of each time allocation, there are only three states for green lights of each phase: increase, constant, and decrease, and only one of them can be selected. The probability sum of the three states is 1 .

$$
\begin{gathered}
P_{i}^{+}=\frac{\left(s_{i}^{+}\right)^{2}}{\left(s_{i}^{+}\right)^{2}+\left(\theta_{i}^{+}\right)^{2}}=\frac{\left(\frac{q_{i}}{q_{i}+t_{g}^{i}}\right)^{2}}{\left(\frac{c_{i}}{q_{i}+t_{g}^{i}}\right)^{2}+\left(\frac{1}{k q_{i}}\right)^{2}} \\
P_{i}^{-}=\frac{\left(s_{i}^{-}\right)^{2}}{\left(s_{i}^{-}\right)^{2}+\left(\theta_{i}^{-}\right)^{2}}=\frac{\left(\frac{t_{g}^{i}}{q_{i}+t_{g}^{i}}\right)^{2}}{\left(\frac{t_{g}^{i}}{q_{i}+t_{g}^{i}}\right)^{2}+\left(k q_{i}\right)^{2}} \\
P_{i}^{*}=1-P_{i}^{+}-P_{i}^{-}
\end{gathered}
$$

where $P_{i}{ }^{+}$is the selection probability of the green time increase in phase $i, P_{i}^{-}$is the selection probability of the green time decrease, and $P_{i}{ }^{*}$ is the selection probability of the green time invariant.

Equations (7) and (8) show that the stronger the stimulus of increasing green time is, and the lower the response threshold of increasing green time is, the greater the probability of increasing green time is. Similarly, the stronger the stimulus of reducing green time is, and the lower the response threshold of reducing green time is, the greater the probability of green time reduction is.

\subsubsection{Task Response}

In the ant colony labor division model, most tasks are expressed in the form of stimulus, and ants decide whether to perform tasks through probability. In the traffic intersection signal timing problem, the probability of task execution is used to determine whether the green time in a phase needs to be adjusted. Let $\beta$ be the lower limit threshold of $P_{i}^{+}$. Only when $P_{i}^{+}$is greater than $\beta$, i.e., $P_{i}^{+}>\beta$ will the green time of the phase be increased. The increment is:

$$
\xi_{i}^{+}=f\left(P_{i}^{+}\right)
$$

In Equation (10), $\xi_{i}^{+}$is a positive correlation function of $P_{i}^{+}$. When the probability of green time increase is greater than the lower limit of the probability of green time increase, 
the green time of this phase increases. The greater the probability of task execution is, the more likely the green time will increase.

Let $\alpha$ be the lower limit threshold of $P_{i}^{-}$. Only when $P_{i}^{-}$is greater than $\alpha$, i.e., $P_{i}^{-}>\alpha$, will the green time of phase $i$ be decreased. The reduction amount is:

$$
\xi_{i}^{-}=f\left(P_{i}^{-}\right)
$$

Similar to Equation (10), in Equation (11), $\xi_{i}^{-}$is a positive correlation function of $P_{i}^{-}$. When the probability of green time reduction is greater than the lower limit of the probability of green time reduction, the green time of the phase decreases. The greater the probability of task execution is, the more likely the green time will decrease.

When $P_{i}^{+}<\beta$ and $P_{i}^{-}<\alpha$, the green time of phase $i$ remains unchanged.

\subsubsection{Algorithm implementation}

According to the previous description of the model, the algorithm implementation process of the model is given, as shown in Figure 1. The process is described as follows:

(1) Parameters including phase number $n$, initial green time, scale coefficient $k$, maximum iteration number $\mathrm{N}$, etc., are initialized.

(2) The initial number of iterations is set to 1 .

(3) According to Equations (3)-(8), the environmental stimulus, response threshold, and probability of task execution of each phase are calculated.

(4) If $P_{i}^{+}$is greater than $\beta$ or $P_{i}^{-}$is greater than $\alpha$, jump to (5), otherwise, jump to (6).

(5) According to Equation (10) or (11), the change amount of green time is calculated and then the green time of each phase is updated.

(6) According to Equations (12)-(17), the delay time of vehicles $D_{i}$, stopping times of vehicles $H_{i}$, and the road traffic capacity $Q_{i}$ are calculated.

(7) The value of evaluation function $F(x, c)$ is calculated according to Equation (18).

(8) Update the $\min F(x, c)$, and the corresponding optimal solution of green time.

(9) Increase the number of iterations by 1.

(10) Judge whether the maximum number of iterations has been reached. If so, jump to (11); otherwise, jump to (3).

(11) Output the optimal solution.

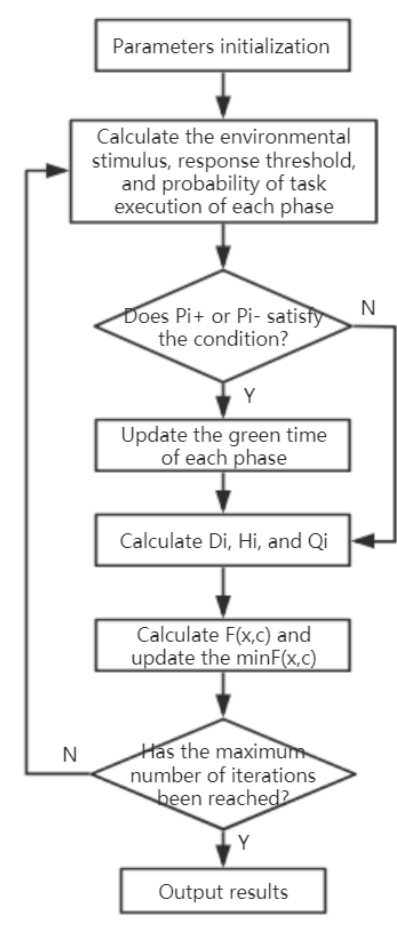

Figure 1. The algorithm calculation process. 


\section{Experiment and Analysis}

\subsection{Design of the Signal Phase}

There are many types of intersections in the city, including intersection, roundabout, and interchange. Intersection includes T-shaped intersection, Y-shaped intersection, Xshaped intersection, cross-shaped intersection, and so on. For the sake of simplification, only the most common cross-shaped intersections in daily life are considered in this paper, of which shapes are shown in Figure 2. The intersection points to the different directions of the road, and the vehicles drive in different directions, which leads to the intersection become a traffic accident-prone point, known as the conflict point [29]. In Figure 2, the east, west, south, and north directions of the intersection are divided into left, straight and right lanes, and vehicles drive in the corresponding lanes.

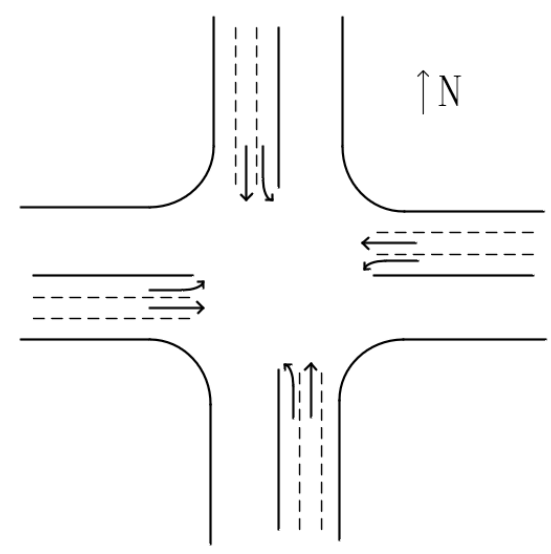

Figure 2. A crossroad.

The phase scheme of the traffic signal lamp includes the two-phase scheme, the threephase scheme, the four-phase scheme and so on. In the four-phase scheme, the special left-turn phase is set in each direction. Compared with the two-phase scheme and the three-phase scheme, the conflict points are effectively eliminated and the traffic efficiency is improved when the road is busy, so this paper discusses the four-phase scheme, and its schematic diagram is shown in Figure 3.

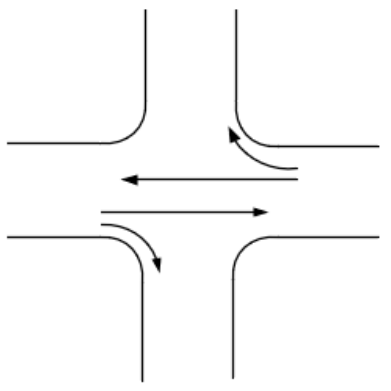

Phase 1

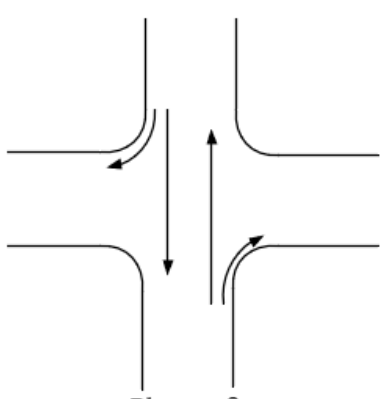

Phase 3

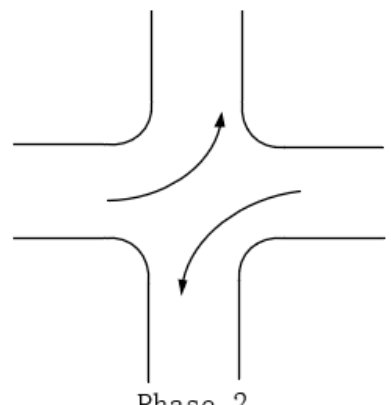

Phase 2

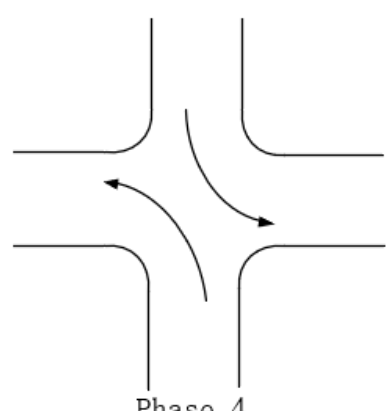

Phase 4

Figure 3. The four-phase scheme. 


\subsection{Experiment Data}

The data used in this paper come from "2020 China University computer competition - Huawei cloud big data challenge", which describes the traffic situation at the intersection of Wuhe Avenue and Zhangheng Road in Longgang, Shenzhen. It intercepts the traffic flow at the intersection from $7 \mathrm{am}$ to $11 \mathrm{pm}$ on 12 January, 2020, and sets every five minutes as a unit time. Due to the excessive number of straight cars in all directions of the intersection, in order to eliminate the conflict points and reduce the possibility of accidents, the four-phase scheme is selected. After processing, the traffic flow at the intersection on that day is shown in Figure 4.

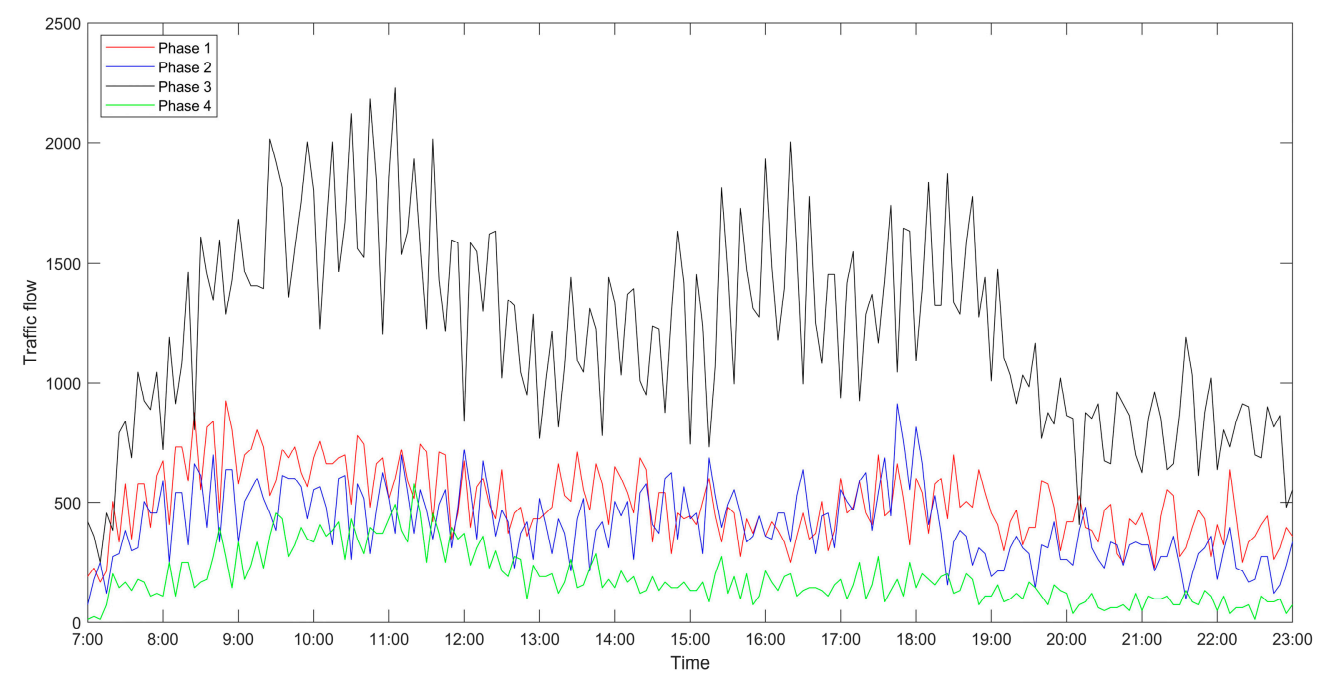

Figure 4. Traffic flow at the intersection of Wuhe Avenue and Zhangheng Road.

\subsection{Evaluation Index}

Under the premise of ensuring traffic safety, the main purpose of signal timing at the intersection is to maximize the efficiency of the intersection, not only to make full use of road traffic resources, but also to protect the rights and interests of road users. Therefore, the average delay time of vehicles, the average number of stops of vehicles and the traffic capacity are selected as evaluation indexes to optimize the traditional traffic signal timing scheme.

For intersections, the vehicle delay formula on the Webster algorithm has been widely used in the field of traffic control [30]. The specific formulas are as follows [31]:

$$
\begin{gathered}
D_{i}=\frac{C\left(1-\lambda_{i}\right)^{2}}{2\left(1-\lambda_{i} y_{i}\right)}+\frac{y_{i}^{2}}{2 q_{i}\left(1-y_{i}\right)} \\
T=\frac{\sum_{i=1}^{n} D_{i} q_{i}}{\sum_{i=1}^{n} q_{i}}
\end{gathered}
$$

where $D_{i}$ is the delay time of vehicles in phase $i, T$ is the average delay time of vehicles in a cycle, $C$ is the total duration of the cycle, $\lambda_{i}$ is the green ratio which is the ratio of the effective green time of phase $i$ to the total duration of the cycle, $y_{i}$ is the degree of traffic flow saturation which is the traffic flow in phase $i$ to the saturated traffic flow, $q_{i}$ is the traffic flow in phase $i$.

$$
H_{i}=0.9 \frac{1-\lambda_{i}}{1-y_{i}}
$$

where $H_{i}$ is the number of stops of vehicles in phase $i$.

$$
H=\frac{\sum_{i=1}^{n} H_{i} q_{i}}{\sum_{i=1}^{n} q_{i}}
$$


where $H$ is the average number of stops of vehicles in phase $i$.

$$
Q_{i}=S_{i} \lambda_{i}=S_{i} \frac{x_{i}}{C}
$$

where $Q_{i}[31]$ is the traffic capacity in phase $i, S_{i}$ is the traffic saturation flow rate in phase $i$, $x_{i}$ is the effective green time of phase $i$.

$$
Q=\sum_{i}^{n} Q_{i}
$$

where $Q[29]$ is the total traffic capacity of the intersection.

\subsection{Experiment}

The traffic signal timing scheme proposed in this paper will be compared with the traditional fixed timing scheme. In the traditional fixed timing scheme, the cycle length and the green time of each phase in a day remain unchanged. The scheme proposed in this paper will judge whether the cycle length and green time should be adjusted according to the number of vehicles passing through the intersection. The solution optimization model is given in reference [32]:

$$
\min F(x, c)=\frac{\sum_{i=1}^{n}\left(K_{1} D_{i}+K_{2} H_{i}\right)}{\sum_{i=1}^{n} K_{3} Q_{i}}
$$

It is assumed that the saturated traffic flow at each phase of the intersection is 1000 $\mathrm{pcu} / \mathrm{h}, 1000 \mathrm{pcu} / \mathrm{h}, 2500 \mathrm{pcu} / \mathrm{h}$ and $600 \mathrm{pcu} / \mathrm{h}$. The time of the amber is $3 \mathrm{~s}$, the change interval is $5 \mathrm{~s}$, and the start-up delay is $3 \mathrm{~s}$. The shortest and longest green time is $20 \mathrm{~s}$ and $90 \mathrm{~s}$, and the initial green time is $40 \mathrm{~s} . f\left(\mathrm{Pi}^{+}\right)$is an exponential function with e as the base and $\mathrm{Pi}^{+}$as the exponent. $f\left(\mathrm{Pi}^{-}\right)$is an exponential function with e as the base and $\mathrm{Pi}^{-}$as the exponent. The maximum number of iterations $\mathrm{N}$ is set to 50 . The simulation calculation environment is described as follows: MATLAB language is used to complete the implementation of the algorithm in this paper, and the experiment is carried out on a $\mathrm{PC}$ with a $2.5 \mathrm{GHz} \mathrm{CPU}$ and $8 \mathrm{~GB}$ memory.

The algorithm took $1.99 \mathrm{~s}$ to execute. The results of the traditional fixed timing scheme and the optimized results using the ant colony labor division model proposed in this paper are shown in Tables 1-3. The result comparison diagrams are shown in Figures 5-7.

Table 1. Comparison of the average delay time of vehicles at intersections.

\begin{tabular}{cccc}
\hline Phase i & $\begin{array}{c}\text { Traditional Fixed Timing } \\
\text { Scheme/s }\end{array}$ & $\begin{array}{c}\text { Ant Colony Labor Division } \\
\text { Model/s }\end{array}$ & Relative Change/\% \\
\hline Phase 1 & 6.65 & 6.04 & -9.06 \\
Phase 2 & 6.27 & 5.59 & -10.71 \\
Phase 3 & 6.63 & 5.97 & -9.95 \\
Phase 4 & 6.15 & 5.23 & -14.83 \\
The average of the intersections & 6.53 & 5.86 & -10.25 \\
\hline
\end{tabular}

Table 2. Comparison of the average number of stops of vehicles at intersections.

\begin{tabular}{cccc}
\hline Phase i & $\begin{array}{c}\text { Traditional Fixed Timing } \\
\text { Scheme }\end{array}$ & $\begin{array}{c}\text { Ant Colony Labor Division } \\
\text { Model }\end{array}$ & Relative Change/\% \\
Phase 1 & 1.0780 & 0.8253 & -23.44 \\
Phase 2 & 0.8871 & 0.6885 & -22.38 \\
Phase 3 & 1.0683 & 0.8071 & -24.44 \\
Phase 4 & 0.9831 & 0.6602 & -32.84 \\
The average of the intersections & 1.0314 & 0.7782 & -24.55 \\
\hline
\end{tabular}


Table 3. Comparison of traffic capacity at intersections.

\begin{tabular}{|c|c|c|c|}
\hline Phase i & $\begin{array}{c}\text { Traditional Fixed Timing } \\
\text { Scheme } / \text { pch } \cdot h^{-1}\end{array}$ & $\begin{array}{c}\text { Ant Colony Labor Division } \\
\text { Model/pch } \cdot h^{-1}\end{array}$ & Relative Change/\% \\
\hline Phase 1 & 6073 & 6329 & +4.23 \\
\hline Phase 2 & 6073 & 6252 & +2.95 \\
\hline Phase 3 & 15,181 & 15,832 & +4.28 \\
\hline Phase 4 & 3644 & 3660 & +0.45 \\
\hline The intersection traffic capacity & $30,969.76$ & $32,072.55$ & +3.56 \\
\hline
\end{tabular}

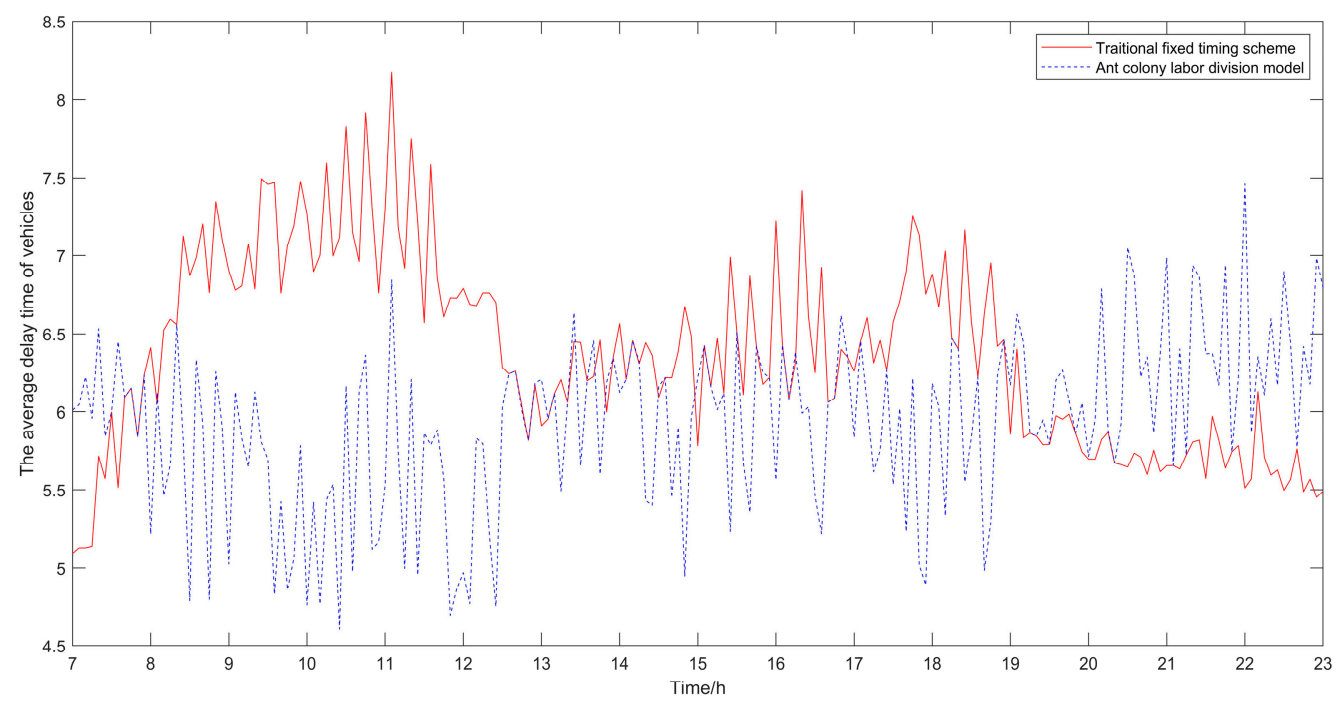

Figure 5. Comparison diagram of average vehicle delay time.

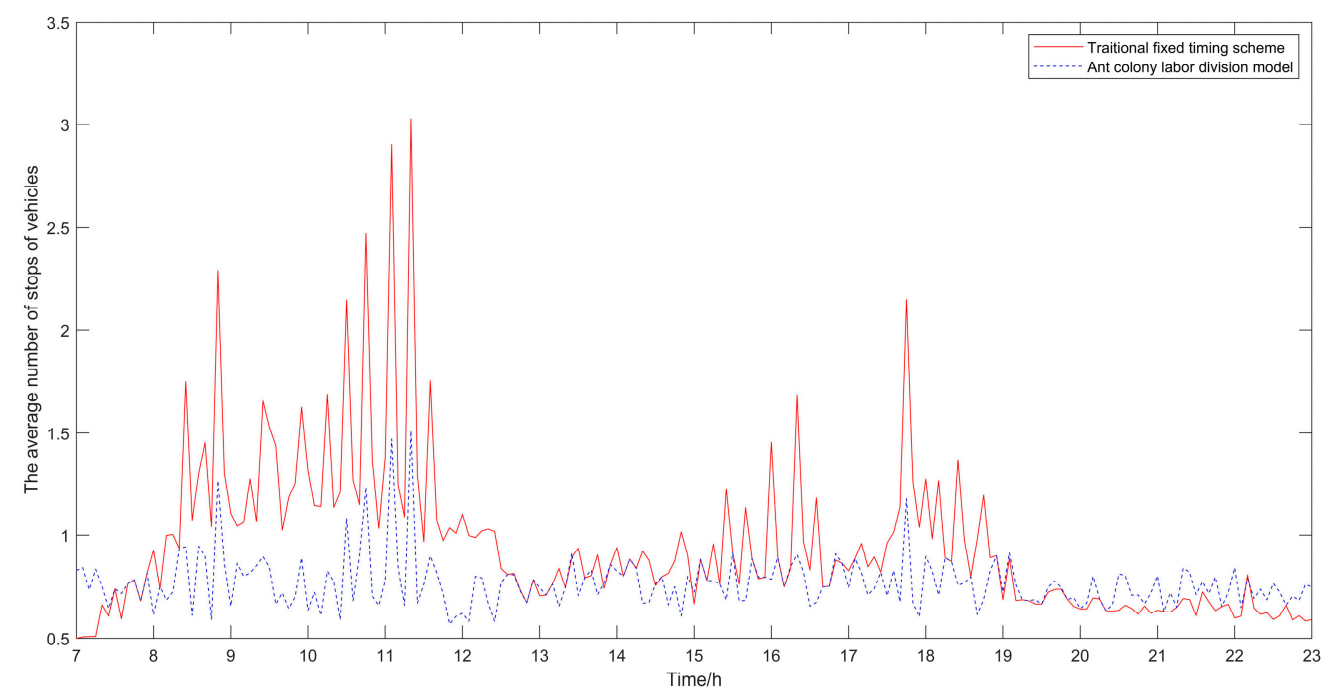

Figure 6. Comparison diagram of the average number of stops of vehicles. 


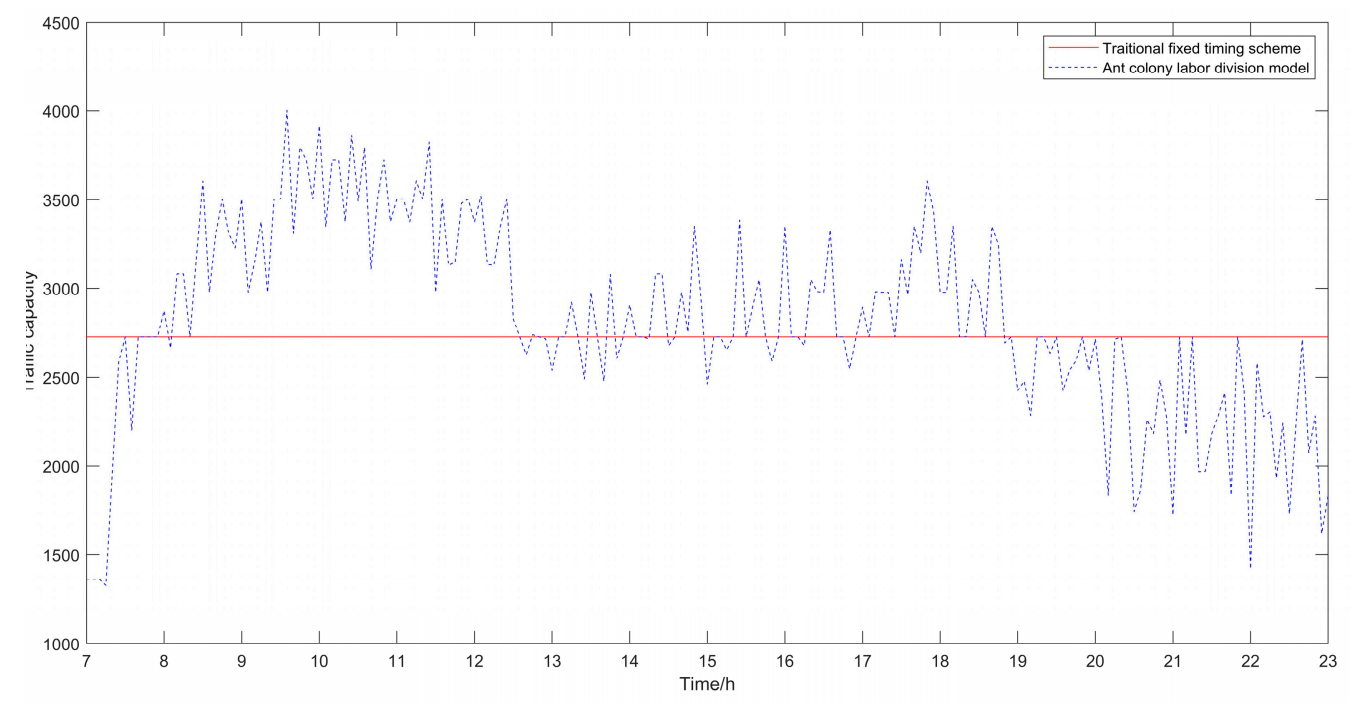

Figure 7. Comparison diagram of traffic capacity at intersections.

\subsection{Result Analysis}

It can be seen from Tables 1-3 that compared with the traditional fixed timing scheme, the scheme optimized by the ant colony labor division model has significant improvement in the delay time of vehicles and the number of stops. The average delay time of vehicles in the traditional fixed timing scheme is $6.53 \mathrm{~s}$, and in the optimized scheme is $5.86 \mathrm{~s}$, which is $10.25 \%$ lower than that in the traditional fixed-timing scheme. The average number of stops in the traditional fixed timing scheme is 1.0314 , and after optimization, it is 0.7782 , which is $24.55 \%$ lower than that in the traditional fixed timing scheme. It can be seen that in terms of the average delay time of vehicles and the average number of stops, the results of the proposed optimization algorithm proposed in this paper are obviously better than those of the traditional fixed timing scheme.

Besides, the results of the optimized algorithm also have a corresponding improvement in the traffic capacity, but the improvement is not significant. In order to better analyze the changes before and after optimization, the comparison diagrams of the average vehicle delay time, the average number of stops of vehicles and the traffic capacity at intersections are shown (as shown in Figures 4-6). As can be seen from Figure 6, the traffic capacity has been greatly improved during 9:00-12:00 on that day, and from 14:00 to 19:00. Although the data fluctuates up and down, the traffic capacity is improved. The traffic capacity, however, decreased during 7:00-8:00 and 19:00-23:00. Combined with Equations (16) and (17), and the trend analysis of traffic flow in Figure 3, it is found that the traffic capacity of the optimized algorithm is related to the effective green time of the phase. When the traffic flow of this phase is very low, according to the algorithm, the green time of this phase will be decreased. Correspondingly, the effective green time will also be decreased, and the green ratio will be decreased, leading to the decrease of the traffic capacity of the intersection. However, from the point of the traffic flow saturation in the intersection, there is no over-saturation state in the whole day, nor does it exceed the maximum capacity of the intersection. It can be said that this phenomenon has very little impact on the traffic operation efficiency of the intersection.

From Figures 5-7, it can be seen that in the period of busy traffic flow, such as 8:00-12:00 and 17:00-19:00, the average delay time and the average number of stops of vehicles after optimization are significantly reduced, and the intersection capacity is also significantly improved. According to calculation, during the period of 8:00-12:00, compared with the fixed timing scheme, the average delay time of vehicles is reduced by $22.01 \%$ in the timing scheme optimized by the ant colony labor division model, the average number of stops of vehicles is reduced by $40.92 \%$, and the road capacity is increased by $24.37 \%$. During the period of 17:00-19:00, the average delay time of vehicles is reduced by $12.46 \%$, 
the average number of stops of vehicles is reduced by $25.29 \%$, and the road capacity is increased by $9.92 \%$. However, in the period after 20:00, due to the small traffic flow, the possibility of congestion is low, and the optimization effect of the algorithm is not obvious.

In general, the scheme proposed in this paper is more suitable for the situation of heavy traffic flow, and the greater the traffic flow, the better the improvement effect of the scheme on vehicle delay time, number of stops and road capacity will be. Ant colony labor division model can alleviate traffic congestion at intersections to a certain extent and improve the utilization rate of roads.

\section{Conclusions}

In the problem of traffic intersection green time allocation, the traffic flow in each phase changes dynamically. In order to relieve the congestion at the intersection, the traffic signal green time of each phase should change dynamically with the traffic flow, rather than remain unchanged.

As a new optimization model, the ant colony labor division model has been widely applied to the dynamic task assignment problem because of its flexibility. In this paper, the ant colony labor division model is applied to the problem of traffic green time allocation at traffic intersections. The simulation experiment results show that compared with the fixed timing scheme, the proposed timing scheme can reduce the average delay time of vehicles by $10.25 \%$ and the average number of stops by $24.55 \%$, and it can increase the road capacity by $3.56 \%$. These data proved that the effect of this scheme is better than the traditional fixed timing scheme, and it can better alleviate the traffic pressure at the intersections during rush hours.

Due to the strong real-time uncontrollability of the traffic situation at the intersection, the next research direction is to further optimize the precision of the model, and try to combine it with the video monitoring, which can obtain the traffic flow and other information, as well as extend the application range from the single intersection to urban trunk lines, and even extended to larger urban traffic network.

Author Contributions: X.F. contributed to the conception of the study. H.G. and H.C. performed the experiment and the analysis with constructive discussions; W.C. and Z.W. performed the data analyses. All authors wrote and revised the manuscript. All authors have read and agreed to the published version of the manuscript.

Funding: This research received no external funding.

Institutional Review Board Statement: Not applicable.

Informed Consent Statement: Not applicable.

Data Availability Statement: The study used the open data.

Acknowledgments: The authors would like to thank the editor and the reviewers for the helpful suggestions and kind help.

Conflicts of Interest: The authors declare no conflict of interest.

\section{References}

1. Dorigo, M. Optimization, Learning and Natural Algorithma. Ph. D. Thesis, Dipartmento di Elettronica, Politecnico di Milano, Milan, Italy, 1992. (In Italian).

2. Liu, F.; Zhong, S. A Review of Foreign Literatures on "swarm intelligence" Research. Stud. Dialectics Nat. 2012, $28,114-117$. (In Chinese) [CrossRef]

3. Kennedy, J.; Eberhart, R.C. Particle swarm optimization. In Proceedings of the IEEE International Conference on Neural Networks, Perth, Australia, 27 November-1 December 1995.

4. Li, X.; Shao, Z.; Qian, J. An Optimizing Method Based on Autonomous Animats: Fish-swarm Algorithm. Syst. Eng. Theory Pract. 2002, 22, 32-38.

5. Karaboga, D. An Idea Based on Honey Bee Swarm for Numerical Optimization; Computers Engineering Department, Engineering Faculty, Erciyes University: Kayseri, Turkey, 2005. 
6. Chen, T.; Li, Q.; Fu, P.; Yang, J.; Xu, C.; Cong, G.; Li, G. Public Opinion Polarization by Individual Revenue from the Social Preference Theory. Int. J. Environ. Res. Public Health 2020, 17, 946. [CrossRef] [PubMed]

7. Ni, X.; Zhou, H.; Chen, W. Addition of an Emotionally Stable Node in the SOSa-SPSa Model for Group Emotional Contagion of Panic in Public Health Emergency: Implications for Epidemic Emergency Responses. Int. J. Environ. Res. Public Health 2020, 17, 5044. [CrossRef] [PubMed]

8. Chen, T.; Wang, Y.; Yang, J.; Cong, G. Modeling Public Opinion Reversal Process with the Considerations of External Intervention Information and Individual Internal Characteristics. Healthcare 2020, 8, 160. [CrossRef] [PubMed]

9. Chen, T.; Li, Q.; Fu, P.; Yang, J.; Xu, C.; Cong, G.; Li, G. Modeling of the Public Opinion Polarization Process with the Considerations of Individual Heterogeneity and Dynamic Conformity. Mathematics 2019, 7, 917. [CrossRef]

10. Chen, W.; Jiang, Z.; Guo, H.; Ni, X. Fall Detection Based on Key Points of Human-Skeleton Using OpenPose. Symmetry 2020, 12, 744. [CrossRef]

11. Chen, T.; Peng, L.; Yin, X.; Rong, J.; Yang, J.; Cong, G. Analysis of User Satisfaction with Online Education Platforms in China during the COVID-19 Pandemic. Healthcare 2020, 8, 200. [CrossRef]

12. Chen, W.; Chen, S.; Guo, H.; Ni, X. Welding flame detection based on color recognition and progressive probabilistic Hough transform. Concurr. Comput. Pract. Exp. 2020, 32, e5815. [CrossRef]

13. Chen, T.; Wu, S.; Yang, J.; Cong, G. Risk Propagation Model and Its Simulation of Emergency Logistics Network Based on Material Reliability. Int. J. Environ. Res. Public Health 2019, 16, 4677. [CrossRef] [PubMed]

14. Chen, T.; Wu, S.; Yang, J.; Cong, G.; Li, G. Modeling of Emergency Supply Scheduling Problem Based on Reliability and Its Solution Algorithm under Variable Road Network after Sudden-Onset Disasters. Complexity 2020. [CrossRef]

15. Xiao, R.; Wang, Y. Research Progress of Self-organized Labor Division in Swarm Intelligence. Inf. Control. $2019,48,129-139$.

16. Zhang, W.; Zhang, B.; Qiu, W. The Use of Ant Colony Model in the Staff Deployment Problem. Adv. Mater. Res. 2011, 317, 1501-1506. [CrossRef]

17. Xiao, R.; Chen, W.; Chen, T. Modeling of Ant Colony's Labor Division for the Multi-Project Scheduling Problem and Its Solution by PSO. J. Comput. Theor. Nanosci. 2012, 9, 223-232. [CrossRef]

18. Wang, Y.; Wang, Y.; Sun, J.; Huang, C.; Zhang, X. A stimulus-response-based allocation method for the circle packing problem with equilibrium constraints. Phys. A Stat. Mech. Appl. 2019, 522, 232-247. [CrossRef]

19. Wang, Y.; Xiao, R. Structure Emergence of Spatial Fidelity Zones in Biosystems. J. Bioinform. Intell. Control 2014, 3, 90-99. [CrossRef]

20. Wu, H.; Li, H.; Xiao, R.; Liu, J. Modeling and simulation of dynamic ant colony's labor division for task allocation of UAV swarm. Phys. A Stat. Mech. Appl. 2018, 491, 127-141. [CrossRef]

21. Xiao, R.; Wu, H.; Hu, L.; Hu, J. A swarm intelligence labor division approach to solving complex area coverage problems of swarm robots. Int. J. Bio Inspired Comput. 2020, 15, 224-238. [CrossRef]

22. Su, H. Study on the Optimization Methods of Signal Timing Parameters of Urban Traffic Control. Ph. D. Thesis, Jilin University, Changchun, China, 2004.

23. Hakim, G.P.N.; Firdausi, A.; Alaydrus, M.; Budiyanto, S. Dynamic Traffic Light Timing Control System using Fuzzy TOPSIS Algorithm. In IOP Conference Series Materials Science and Engineering; IOP: London, UK, 2018; Volume 453.

24. Guo, P.; Lei, D.; Ye, L. An Indefinite Cycle Traffic Light Timing Strategy. Int. J. Adv. Comput. Sci. Appl. 2018, 9, 1-12. [CrossRef]

25. Chen, Y.; Chen, K.; Hsiung, P.A. Dynamic traffic light optimization and Control System using model-predictive control method. In Proceedings of the 2016 IEEE 19th International Conference on Intelligent Transportation Systems (ITSC), Rio de Janeiro, Brazil, 1-4 November 2016; pp. 2366-2371.

26. Qi, A. Research on Timing Optimization of Regional Traffic Signals based on Improved Genetic Algorithm. In Proceedings of the 2018 3rd International Workshop on Materials Engineering and Computer Sciences (IWMECS 2018), Jinan, China, 27-28 January 2018.

27. Bonabeau, E.; Theraulaz, G.; Deneubourg, J. Fixed Response Thresholds and the Regulation of Division of Labor in Insect Societies. Bull. Math. Biol. 1998, 60, 753-807. [CrossRef]

28. Castello, E.; Yamamoto, T.; Nakamura, Y.; Ishiguro, H. Foraging optimization in swarm robotic systems based on an adaptive response threshold model. Adv. Robot. 2014, 28, 1343-1356. [CrossRef]

29. Wang, B. The Research on Signal Timing Algorithm Intersections Based on Ant Colony Algorithm and Fuzzy Control Theory; Jilin University: Changchun, China, 2018.

30. Yang, P.; Zhang, S. Traffic Management and Control; China Communication Press: Beijing, China, 1995.

31. Chen, X.; Qian, D.; Shi, D. Multi-objective optimization method for signal timing of urban traffic intersection. Mod. Electron. Tech. 2016, 39, 19-22.

32. He, J. Traffic Signal Timing Optimization Based on Ant Colony Algorithm; Shanxi University of Science and Technology: Taiyuan, China, 2012. 